\title{
Fuzzy Finite Element based Solution of Uncertain Static Problems of Structural Mechanics
}

\author{
Diptiranjan Behera \\ Department of Mathematics National Institute of \\ Technology, Rourkela \\ Odisha-769008, India
}

\author{
S. Chakraverty \\ Department of Mathematics National Institute of \\ Technology, Rourkela \\ Odisha-769008, India
}

\begin{abstract}
Fuzzy finite element analysis for static displacements of beam structures with fuzzy forces is considered in this paper. The material properties of the beams are taken as crisp. Fuzzy finite element analysis of static problem for the above structures converts the problem into fuzzy system of linear equations. As such the coefficient matrix and the right hand side vector become crisp and fuzzy respectively. Here, a new method is proposed to solve the fuzzy system of linear equations. Numerical results for the beam structures are presented to illustrate the computational aspects of the developed method. The results obtained by the proposed method are compared with the existing solution method.
\end{abstract}

\section{Keywords}

Triangular fuzzy number, Fuzzy system of linear equations, Fuzzy finite element method, Beam.

\section{INTRODUCTION}

In the last few decades for various scientific and engineering problems finite element method has become a more powerful tool for solving the complex systems. In this method the complicated structures/domains are discretized into small finite elements, giving the element wise behavior. Assembling together for all the elements and applying the respective conditions, it gives the output. The system parameters involved in the traditional finite element method such as mass, geometry, material properties, external loads, or boundary conditions are considered as crisp or defined exactly. But, rather than the particular value we may have only the vague, imprecise and incomplete information about the variables and parameters being a result of errors in measurement, observations, experiment, applying different operating conditions or it may be maintenance induced error, etc. which are uncertain in nature. Basically these uncertainties can be modeled through probabilistic approach, interval analysis and fuzzy theory.

In probabilistic practice, the variables of uncertain nature are assumed as random variables with joint probability density functions. If the structural parameters and the external load are modeled as random variables with known probability density functions, the response of the structure can be predicted using the theory of probability and stochastic processes [1]. Also the probabilistic concept is already well established for the extension of the deterministic finite element method towards uncertain assessment. This has led to a number of probabilistic and stochastic finite element procedures [2,3]. Unfortunately, probabilistic methods are not able to deliver reliable results at the required precision without sufficient experimental data. It may be due to the probability density functions involved in it. As such in the recent decades, interval analysis and fuzzy theory are becoming powerful tools for many real life applications. In these approaches, the uncertain variables and parameters are represented by interval and fuzzy numbers, vectors or matrices.

Various aspects of interval analysis along with applications are explained by [4]. If only incomplete information is available, it is possible to establish the minimum and maximum favorable response of the structures using interval analysis or convex models [5, 6]. Moreover structural analysis with interval parameters using interval based approach has been studied by various authors $[7,8,9]$.

Fuzzy set theoretical concept was developed by [10] which is further used in the uncertain analysis of structures in a wide range. As discussed above, if the structural parameters and the external loads are described in imprecise terms, then fuzzy theory can be applied. As such [11] fuzzy logic for the numerical modeling of engineering problems has been done. An optimization algorithm is developed for fuzzy properties [12] based on response surface for the calculation of fuzzy envelope and fuzzy response functions of models. Fuzzy structural analysis using $\alpha$-level optimization is excellently studied by [13]. The transformation method has been applied for the simulation and analysis of systems with uncertain parameters [14]. Also an important book is written by [15] in which applications of fuzzy arithmetic into engineering problems are described. Fuzzy behavior of mechanical systems with uncertain boundary conditions is investigated by [16]. Nonlinear membership function for fuzzy optimization of mechanical and structural systems is discussed in [17]. When the Finite Element Method (FEM) is described with fuzzy theory it is then known as Fuzzy Finite Element Method (FFEM).

Recently various generalized model of uncertainty have been applied to finite element method to solve the structural problems with fuzzy parameters. Although FEM for structural problems [18] is well known and there exits large number of papers related to this. As such few papers that are related to fuzzy FEM are discussed here. Fuzzy finite element approach is applied to describe structural systems with imprecisely defined parameters in an excellent way by [19]. The fuzzy finite element analysis technique [20] to describe the static analysis of structures which is based on interval computation. Both fuzzy static and dynamic analysis of structures is explained by [21] using fuzzy finite element approach. Vertex method and VAST software is used in it for the fuzzy finite element analysis. Also [22] derived fuzzy finite element method for smart structures. Fuzzy finite element method is formulated by [23] for mechanics problems. In [24] fuzzy arithmetical approach is used for the solution of finite element problems with fuzy parameters. Very recently $[25,26]$ investigated the structural problems with fuzzy parameters. They have used an interesting approach viz. High Dimensional Model Representation (HDMR) along with FEM 
is for the analysis. Both static and dynamic responses of structures is explained by [27] using FFEM with HDMR.

The design and analysis of many engineering problems require the solution of linear systems of equations. For example, the finite element formulation of equilibrium and steady state problems lead to a set of simultaneous algebraic linear equations. Accordingly FFEM converts the problem to a Fuzzy System of Linear Equations (FSLE) [28, 29] or Fully Fuzzy System of Linear Equations (FFSLE) [30-32] for the static analysis of structural problems. There is a difference between fuzzy linear system and fully fuzzy linear system. The coefficient matrix is treated as crisp in the fuzzy linear system, but in the fully fuzzy linear system all the parameters and variables are considered to be fuzzy numbers. Various solution methods have been proposed for the solution of FFSLE [33] and applied in structural mechanics problems. Recently [34] has been developed a method to find finite element solution of a stepped rectangular bar in presence of fuzziness in material properties.

As such it is an important issue to develop mathematical models and numerical techniques that would appropriately treat the general fuzzy or fully fuzzy linear systems because subtraction and division of fuzzy numbers are not the inverse operations of addition and multiplication respectively. So, this is an important area of research in the recent years. This paper targets to propose new methods for fuzzy and fully fuzzy system of linear equations and applied those methods to the analysis of structural problems using FFEM. In the following sections first preliminaries is discussed. Then, a new solution method for fuzzy system of linear equations is proposed. Next, numerical examples of beam with various types of uncertain forces are discussed using fuzzy finite element method to find fuzzy static responses. Lastly conclusions are drawn.

\section{PRILIMINARIES}

In the following paragraph some definitions related to the present work are given $[35,36,38,39]$.

\section{Definition 2.1 Fuzzy number}

A fuzzy number $\tilde{U}$ is convex normalised fuzzy set $\tilde{U}$ of the real line $R$ such that

$$
\left\{\mu_{\tilde{U}}(x): R \rightarrow[0,1], \forall x \in R\right\}
$$

where, $\mu_{\tilde{U}}$ is called the membership function of the fuzzy set and it is piecewise continuous.

\section{Definition 2.2 Triangular fuzzy number (TFN)}

A triangular fuzzy number $\tilde{U}$ is a convex normalized fuzzy set $\tilde{U}$ of the real line $R$ such that

i. there exists exactly one $x_{0} \in R$ with $\mu_{\tilde{U}}\left(x_{0}\right)=1\left(x_{0}\right.$ is called the mean value of $\tilde{U}$ ), where $\mu_{\tilde{U}}$ is called the membership function of the fuzzy set.

ii. $\mu_{\tilde{U}}(x)$ is piecewise continuous.

Let us consider an arbitrary triangular fuzzy number $\tilde{U}=(a, b, c)$. The membership function $\mu_{\tilde{U}}$ of $\tilde{U}$ may be define as follows

$$
\mu_{\tilde{U}}(x)= \begin{cases}0, & x \leq a \\ \frac{x-a}{b-a}, & a \leq x \leq b \\ \frac{c-x}{c-b}, & b \leq x \leq c \\ 0, & x \geq c .\end{cases}
$$

The triangular fuzzy number $\tilde{U}=(a, b, c)$ can be represented with an ordered pair of functions through $\alpha$-cut as $[\underline{u}(\alpha), \bar{u}(\alpha)]=[(b-a) \alpha+a,-(c-b) \alpha+c]$ where $\alpha \in[0,1]$. This satisfies the following requirements

i. $\underline{u}(\alpha)$ is a bounded left continuous non-decreasing function over $[0,1]$.

ii. $\bar{u}(\alpha)$ is a bounded right continuous non-increasing function over $[0,1]$.

iii. $\underline{u}(\alpha) \leq \bar{u}(\alpha), 0 \leq \alpha \leq 1$.

Definition 2.3 Fuzzy arithmetic

Fuzzy numbers may be transformed into an interval through $\alpha$-cut approach. So, for any arbitrary fuzzy number $\tilde{x}=[\underline{x}(\alpha), \bar{x}(\alpha)], \tilde{y}=[\underline{y}(\alpha), \bar{y}(\alpha)]$ and scalar $k$, we have the interval based fuzzy arithmetic as

i. $\quad \tilde{x}=\tilde{y}$ if and only if $\underline{x}(\alpha)=\underline{y}(\alpha)$ and $\bar{x}(\alpha)=\bar{y}(\alpha)$

ii. $x+y=[\underline{x}(\alpha)+\underline{y}(\alpha), \bar{x}(\alpha)+\bar{y}(\alpha)]$

iii. $\tilde{x}-\tilde{y}=[\underline{x}(\alpha)-\bar{y}(\alpha), \bar{x}(\alpha)-\underline{y}(\alpha)]$

$\tilde{x} \times \tilde{y}=[\min (\underline{x}(\alpha) \times \underline{y}(\alpha), \underline{x}(\alpha) \times \bar{y}(\alpha), \bar{x}(\alpha) \times y(\alpha), \bar{x}(\alpha) \times \bar{y}(\alpha)$,

iv. $\max (\underline{x}(\alpha) \times \underline{y}(\alpha), \underline{x}(\alpha) \times \bar{y}(\alpha), \bar{x}(\alpha) \times \underline{y}(\alpha), \bar{x}(\alpha) \times \bar{y}(\alpha)]$

v. $k \tilde{x}= \begin{cases}{[k \bar{x}(\alpha), k \underline{x}(\alpha)],} & k<0, \\ {[k \underline{x}(\alpha), k \bar{x}(\alpha)],} & k \geq 0 .\end{cases}$

The equation of motion with fuzzy parameters of the structures obtained by FFEM reduces to fuzzy system of linear equations. As such method for fuzzy system of linear equations is proposed here to find the fuzzy static response.

\section{PROPOSED METHOD FOR FUZZY SYSTEM OF LINEAR EQUATIONS}

The $n \times n$ fuzzy system of linear equations may be written as

$$
\begin{gathered}
a_{11} \tilde{x}_{1}+a_{12} \tilde{x}_{2}+\cdots+a_{1 n} \tilde{x}_{n}=\tilde{b}_{1} \\
a_{21} \tilde{x}_{1}+a_{22} \tilde{x}_{2}+\cdots+a_{2 n} \tilde{x}_{n}=\tilde{b}_{2} \\
\vdots \\
a_{n 1} \tilde{x}_{1}+a_{n 2} \tilde{x}_{2}+\cdots+a_{n n} \tilde{x}_{n}=\tilde{b}_{n}
\end{gathered}
$$

In matrix notation the above system may be written as

$$
[A]\{\tilde{X}\}=\{\tilde{b}\},
$$

where, the coefficient matrix $[A]=\left(a_{k j}\right), 1 \leq k \leq n, j \leq n$ is a crisp real $n \times n$ matrix, $\{\tilde{b}\}=\left\{\tilde{b}_{k}\right\}, 1 \leq k$ is a column vector of triangular fuzzy number and $\{\tilde{X}\}=\left\{\tilde{x}_{j}\right\}$ is the vector of fuzzy unknown.

The above system, $[A]\{\tilde{X}\}=\{\tilde{b}\}$, can be written as

$$
\sum_{j=1}^{n} a_{k j} \tilde{x}_{j}=\tilde{b}_{k}, \text { for } k=1,2, \cdots, n \text {. }
$$


As per the parametric form we may write the real fuzzy unknown and the right hand real fuzzy number vector as

$$
\tilde{x}_{j}=\tilde{x}_{j}(\alpha)=\left[\underline{x}_{j}(\alpha), \bar{x}_{\mathrm{j}}(\alpha)\right]
$$

and

$$
\tilde{b}_{k}=\tilde{b}_{k}(\alpha)=\left[\underline{b}_{k}(\alpha), \bar{b}_{k}(\alpha)\right] .
$$

where, $\alpha \in[0,1]$.

Substituting these expressions in (2), we have

$$
\sum_{j=1}^{n} a_{k j}\left[\underline{x}_{j}(\alpha), \bar{x}_{\mathrm{j}}(\alpha)\right]=\left[\underline{b}_{k}(\alpha), \bar{b}_{k}(\alpha)\right] .
$$

In the following a new method is proposed for solving the fuzzy system of linear equations as defined in (1).

Through $\alpha$-cut form (2) can be represented as

$$
\sum_{j=1}^{n} a_{k j} \tilde{x}_{j}(\alpha)=\tilde{b}_{k}(\alpha)
$$

Now the 1 - cut of the system (4) is solved possibly. The 1cut system of (4) can be represented as below

$$
\sum_{j=1}^{n} a_{k j}(1) \tilde{x}_{j}(1)=\tilde{b}_{k}(1)
$$

Above can be equivalently represented as

$$
\sum_{j=1}^{n} a_{k j}\left[\underline{x}_{j}(1), \bar{x}_{j}(1)\right]=\left[\underline{b}_{k}(1), \bar{b}_{k}(1)\right] \text {. }
$$

$\tilde{x}_{j}(1)$ is now obtained by solving (5). Here $\tilde{x}_{j}(1)$ is known as the core of $\tilde{x}_{j}(\alpha)$. It is worth mentioning that (1) in general (for triangular fuzzy number matrix) converted to a crisp system for finding the core solution. That means (6) is a crisp system with triangular fuzzy number. So we have $\underline{b}_{k}(1)=\bar{b}_{k}(1)$ and $\underline{x}_{j}(1)=\bar{x}_{j}(1)$.

Now the solution vector $\tilde{x}_{j}(\alpha)$ can be represented for triangular fuzzy number as

$$
\left[\tilde{x}_{j}(1)-\delta(\alpha), \tilde{x}_{j}(1)+\gamma(\alpha)\right]
$$

where $\delta(\alpha)$ and $\gamma(\alpha)$ are left and right spreads of the solution vector for $\alpha \in[0,1]$.

As such (1) can be expressed for triangular fuzzy number solutions as

$$
\sum_{j=1}^{n} a_{k j}\left[\tilde{x}_{j}(1)-\delta(\alpha), \tilde{x}_{j}(1)+\gamma(\alpha)\right]=\left[\underline{b}_{k}(\alpha), \bar{b}_{k}(\alpha)\right] \text {. }
$$

Hence (7) may equivalently be written as the following two equations viz. Eqs. (8) and (9)

$$
\sum_{a_{k j} \geq 0} a_{k j}\left\{\tilde{x}_{j}(1)-\delta(\alpha)\right\}+\sum_{a_{k j}<0} a_{k j}\left\{\tilde{x}_{j}(1)+\gamma(\alpha)\right\}=\underline{b}_{k}(\alpha)
$$

and

$$
\sum_{a_{k j} \geq 0} a_{k j}\left\{\tilde{x}_{j}(1)+\gamma(\alpha)\right\}+\sum_{a_{k j}<0} a_{k j}\left\{\tilde{x}_{j}(1)-\delta(\alpha)\right\}=\bar{b}_{k}(\alpha)
$$

Finally (8) and (9) are solved to find the left and right spread viz. $\delta(\alpha)$ and $\gamma(\alpha)$ of the solution vector. Hence for triangular fuzzy number system the solution vector can be written as $\left[\tilde{x}_{j}(1)-\delta(\alpha), \tilde{x}_{j}(1)+\gamma(\alpha)\right]$.

\section{NUMERICAL EXAMPLES AND RESULTS}

The proposed method is used here to compute the fuzzy static response of beam structures as shown in the following example problems.

Example 1: Let us consider a beam as shown in Fig. 1. Here vertical displacement and angle of rotations are denoted by $\tilde{u}_{j}$ for $j=1,3,5$ and $\tilde{u}_{j}$ for $j=2,4,6$ respectively. The loads acting on beam are considered as both crisp and triangular fuzzy number for two different cases 1 and 2 to compute the static response. Two different uncertain uniformly distributed loads $\tilde{p}$ and $\tilde{q}$ act on elements 1 and 2 respectively. For each section of the beam Young's modulus, Moment of inertia and length are assumed respectively as $E^{(i)}=2 \times 10^{8} \mathrm{KN} / \mathrm{m}^{2}, \quad I^{(i)}=5 \times 10^{-6} \mathrm{~m}^{4} \quad$ and $l^{(i)}=5 m$ for $i=1,2,3$.

Due to uncertain uniformly distributed load, the fuzzy load vector for element one and two can be written respectively as

$$
\tilde{F}_{1}=\left\{\begin{array}{l}
\tilde{r} l^{(1)} / 2 \\
\tilde{r}\left(l^{(1)}\right)^{2} / 12 \\
\tilde{r} l^{(1)} / 2 \\
-\tilde{r}\left(l^{(1)}\right)^{2} / 12
\end{array}\right\} \text { where } \tilde{r}=-\tilde{p}
$$

and

$$
\tilde{F}_{2}=\left\{\begin{array}{l}
\tilde{s} l^{(2)} / 2 \\
\tilde{s}\left(l^{(2)}\right)^{2} / 12 \\
\tilde{s} l^{(2)} / 2 \\
-\tilde{s}\left(l^{(2)}\right)^{2} / 12
\end{array}\right\} \text { where } \tilde{s}=-\tilde{q} .
$$

Assembling the above, the load vector will be

$$
\tilde{F}=\left\{\begin{array}{l}
\tilde{r} l^{(1)} / 2 \\
\tilde{r}\left(l^{(1)}\right)^{2} / 12 \\
\left(\tilde{r} l^{(1)} / 2\right)+\left(\tilde{s} l^{(2)} / 2\right) \\
\left(-\tilde{r}\left(l^{(1)}\right)^{2} / 12\right)+\left(-\tilde{s}\left(l^{(2)}\right)^{2} / 12\right) \\
\tilde{s} l^{(2)} / 2 \\
-\tilde{s}\left(l^{(2)}\right)^{2} / 12
\end{array}\right\} .
$$

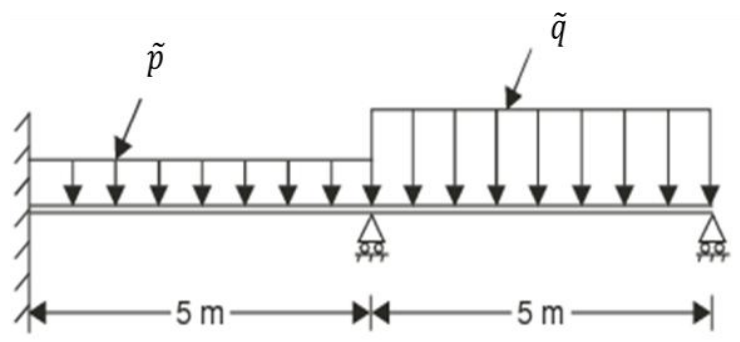

(a) 


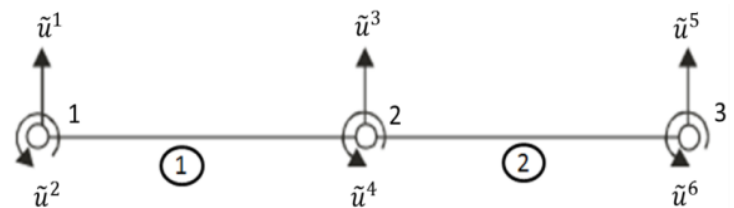

(b)

Fig. 1. Two element discretization of beam with uniformly distributed load

Case 1: The uniformly distributed loads are considered as crisp [37] that is

$$
p=\tilde{p}=12 \mathrm{kN} / \mathrm{m} \text { and } q=\tilde{q}=24 \mathrm{kN} / \mathrm{m} .
$$

One may obtain the static response by finite element analysis of the above as

$$
\begin{aligned}
& \tilde{u}^{(4)}=u^{(4)}=-0.0357142 \mathrm{~m}, \\
& \tilde{u}^{(6)}=u^{(6)}=0.0803571 \mathrm{~m} .
\end{aligned}
$$

Obtained results for crisp parameters are compared with the crisp solution of [37] and found to be similar.

Case 2: Next, the uniformly distributed loads are taken as triangular fuzzy numbers that are

$$
\tilde{p}=(4,12,20) \mathrm{kN} / \mathrm{m} \text { and } \tilde{q}=(20,24,28) \mathrm{kN} / \mathrm{m} \text {. }
$$

Results are shown by solving the governing fuzzy system of linear equations (obtained by using FFEM) in Table 1 .

Table 1: Left and right bounds of fuzzy static response for triangular fuzzy nodal force for Example 1

\begin{tabular}{|c|c|c|c|c|}
\hline$\alpha$ & 0 & 0.2 & 0.8 & 1 \\
\hline$\underline{u}^{4}$ & -0.0506 & -0.0476 & -0.0387 & -0.0357 \\
\hline $\bar{u}^{4}$ & -0.0208 & -0.0238 & -0.0327 & -0.0357 \\
\hline$\underline{u}^{6}$ & 0.0774 & 0.0780 & 0.0798 & 0.0804 \\
\hline $\bar{u}^{6}$ & 0.0833 & 0.0827 & 0.0810 & 0.0804 \\
\hline
\end{tabular}

This problem is also solved by Chakraverty and Behera [38] and Friedman et al. [28] methods. For $\alpha=0.5$ and the obtained results are compared in Table 2.

Table 2: Comparison of results obtained by present, Chakraverty and Behera [38] and Friedman et al. [28] methods for $\alpha=0.5$ (Example 1)

\begin{tabular}{|c|c|c|c|}
\hline$\alpha=0.5$ & $\begin{array}{c}\text { Present } \\
\text { Method }\end{array}$ & $\begin{array}{c}\text { Chakraverty } \\
\text { and Behera } \\
{[38]}\end{array}$ & $\begin{array}{c}\text { Friedman } \\
\text { et al. [28] }\end{array}$ \\
\hline$\underline{u}^{4}$ & -0.0432 & -0.0432 & -0.0432 \\
\hline $\bar{u}^{4}$ & -0.0283 & -0.0283 & -0.0283 \\
\hline$\underline{u}^{6}$ & 0.0789 & 0.0789 & 0.0789 \\
\hline $\bar{u}^{6}$ & 0.0818 & 0.0818 & 0.0818 \\
\hline
\end{tabular}

Example 2: In this example both nodal force $\tilde{p}$ and uniformly distributed load $\tilde{q}$ are acting as shown in Fig. 2 . The beam structure is again discretized in to two elements. For each section of the beam, Young's modulus and moment of inertia are respectively considered as

$$
E^{(i)}=200 \times 10^{6} \mathrm{KN} / \mathrm{m}^{2}, I^{(i)}=24 \times 10^{-6} \mathrm{~m}^{4} .
$$

Length of the first and second elements respectively are $l^{(1)}=4 m$ and $l^{(2)}=6 m$. Due to uncertain nodal force $\tilde{p}$ at node 2 , the nodal force vector can be written as

$$
\tilde{F}_{1}=\left[\begin{array}{llllll}
0 & 0 & \tilde{r} & 0 & 0 & 0
\end{array}\right]^{T} \text { where, } \tilde{r}=-\tilde{p} .
$$

Moreover for uncertain uniform distributed load $\tilde{q}$, the load vector for element one and two may again be written respectively as

$$
\tilde{F}_{2}=\left\{\begin{array}{l}
\tilde{s} l^{(1)} / 2 \\
\tilde{s}\left(l^{(1)}\right)^{2} / 12 \\
\tilde{s} l^{(1)} / 2 \\
-\tilde{s}\left(l^{(1)}\right)^{2} / 12
\end{array}\right\}
$$

and

where $\tilde{s}=-\tilde{q}$.

$$
\tilde{F}_{3}=\left\{\begin{array}{l}
\tilde{s} l^{(2)} / 2 \\
\tilde{s}\left(l^{(2)}\right)^{2} / 12 \\
\tilde{s} l^{(2)} / 2 \\
-\tilde{s}\left(l^{(2)}\right)^{2} / 12
\end{array}\right\}
$$

Accordingly, the assembled load vector may be obtain as

$$
\tilde{F}=\left\{\begin{array}{l}
\tilde{s} l^{(1)} / 2 \\
\tilde{s}\left(l^{(1)}\right)^{2} / 12 \\
\left(\tilde{s} l^{(1)} / 2\right)+\left(\tilde{s} l^{(2)} / 2\right)+\tilde{r} \\
\left.-\tilde{s}\left(l^{(1)}\right)^{2} / 12\right)+\left(\tilde{s}\left(l^{(2)}\right)^{2} / 12\right) \\
\tilde{s} l^{(2)} / 2 \\
-\tilde{s}\left(l^{(2)}\right)^{2} / 12
\end{array}\right\}
$$

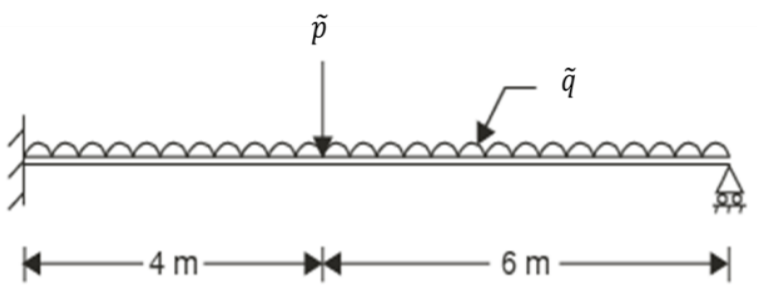

(a)

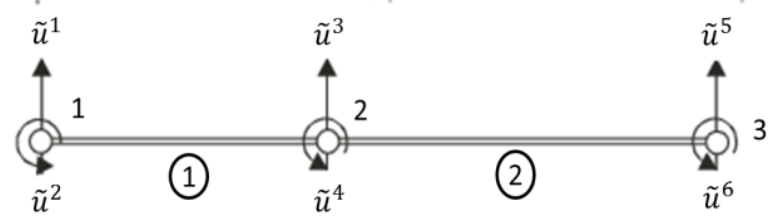

(b)

Fig. 2. Two element discretization of beam with both nodal force and uniform distributed load

Case 1: In this case loads are taken as crisp [37] where, $\tilde{p}=100 \mathrm{kN}$ and $\tilde{p}=20 \mathrm{kN} / \mathrm{m}$. Using finite element analysis one may obtain the static responses as 


$$
\begin{aligned}
& \tilde{u}^{(3)}=u^{(3)}=-0.32733324 \mathrm{~m}, \\
& \tilde{u}^{(4)}=u^{(4)}=-0.07244438 \mathrm{~m}, \\
& \tilde{u}^{(6)}=u^{(6)}=.1368055 \mathrm{~m} .
\end{aligned}
$$

Obtained results for crisp parameters are compared with the crisp solution of [37] and are found to be similar.

Case 2: Here triangular fuzzy loads are taken into consideration. Thus the values of the fuzzy loads are considered as

$$
\tilde{p}=(90,100,110) k N \text { and } \tilde{q}=(10,20,30) \mathrm{kN} / \mathrm{m} .
$$

Fuzzy finite element method is used again and the corresponding governing fuzzy system of linear equations is solved by using the proposed method. Obtained results are shown in Figs. 3 to 5.

It is worth mentioning that this problems is also solved by the methods of $[28,38]$ and the results obtained are exactly same as that of the proposed method. For particular value of $\alpha=0.5$ computed results are tabulated in Table 3 .

Table 3: Comparison of results obtained by present, Chakraverty and Behera [38] and Friedman et al. [28] methods for $\alpha=0.5$ (Example 2)

\begin{tabular}{|c|c|c|c|}
\hline$\alpha=0.5$ & $\begin{array}{c}\text { Present } \\
\text { Method }\end{array}$ & $\begin{array}{c}\text { Chakraverty } \\
\text { and Behera } \\
{[38]}\end{array}$ & $\begin{array}{c}\text { Friedman } \\
\text { et al. [28 ] }\end{array}$ \\
\hline$\underline{u}^{3}$ & -0.3691 & -0.3691 & -0.3691 \\
\hline $\bar{u}^{3}$ & -0.2856 & -0.2856 & -0.2856 \\
\hline$\underline{u}^{4}$ & -0.0825 & -0.0825 & -0.0825 \\
\hline $\bar{u}^{4}$ & -0.0624 & -0.0624 & -0.0624 \\
\hline$\underline{u}^{6}$ & 0.1476 & 0.1476 & 0.1476 \\
\hline $\bar{u}^{6}$ & 0.1260 & 0.1260 & 0.1260 \\
\hline
\end{tabular}

Although the results obtained by all the methods are same but the present procedure is more straight forward and easy to handle. After finding the core solution one may easily find the left and right spread of the solution vector by solving the corresponding crisp systems. So these may give the final solution with the combination of the core solution.

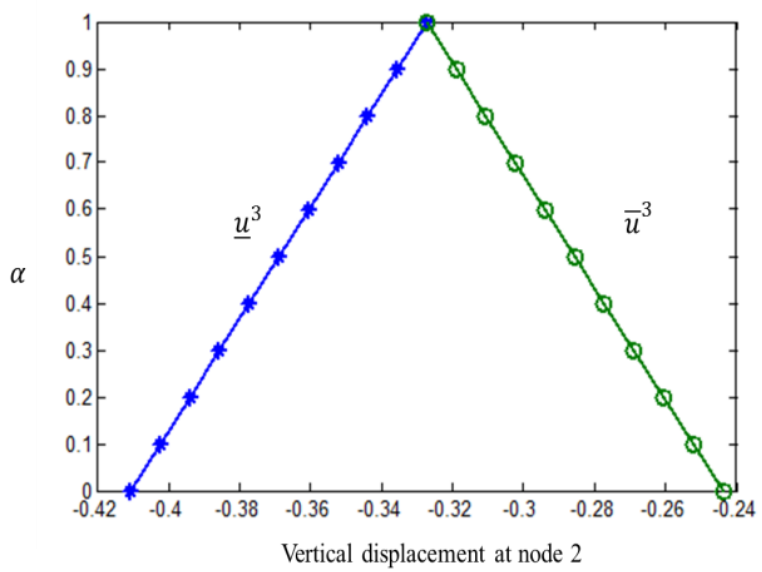

Fig. 3 Left and right bounds of vertical displacement at node 2 for triangular fuzzy forces of Example 2

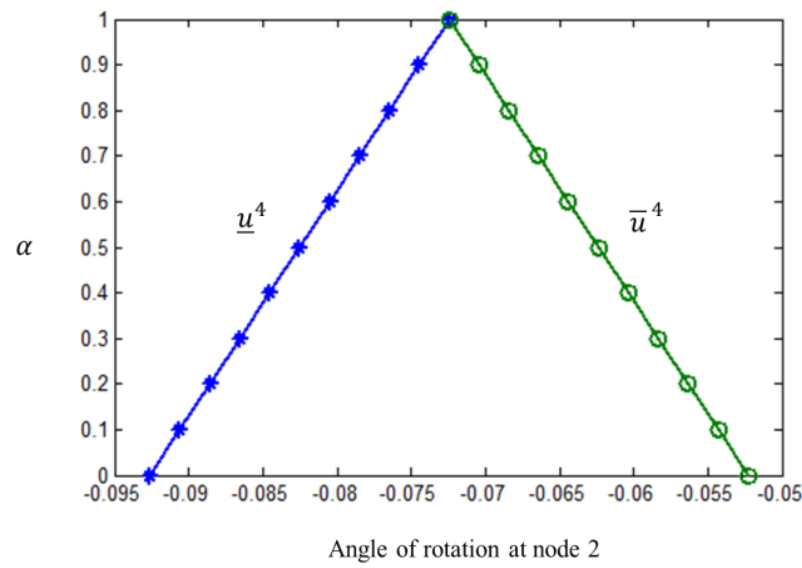

Fig. 4 Left and right bounds of angle of rotation at node 2 for triangular fuzzy forces of Example 2

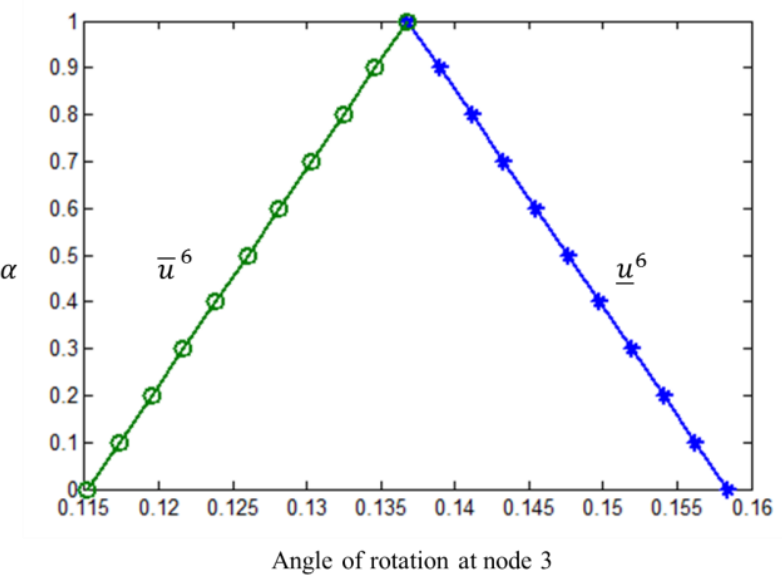

Fig. 5 Left and right bounds of angle of rotation at node 3 for triangular fuzzy forces of Example 2

\section{CONCLUSION}

Static analysis of uncertain beam structures has been studied here using fuzzy finite element method. The related fuzzy system of linear equations is solved using the proposed method which is different from the existing methods to obtain the uncertain static response. The proposed methodology is simple and easy to handle. Investigation presented here may find in real application where the load may not be obtained in term of crisp but a vague value in term of fuzzy is known. Results are depicted in term of plots and tables to show the efficacy of the method.

\section{ACKNOWLEDGMENTS}

This work is financially supported by Board of Research in Nuclear Sciences, Department of Atomic Energy, Government of India.

\section{REFERENCES}

[1] I. Elishakoff, Probabilistic Methods in the Theory of Structures. Wiley, New York, 1983.

[2] A. Haldar and S. Mahadevan, Reliability Assessment Using Stochastic Finite Element Analysis. John Wiley \& Sons, New York, 2000.

[3] C. C. Antonio, and L. N. Hoffbauer, "Uncertainty propagation in inverse reliability-based design of composite structures," Int. J. Mech. Mater. Des., vol. 6, pp. 89-102, 2010. 
[4] R. E. Moore, Methods and Applications of Interval Analysis, SAIM Publication, Philadelphia, 1979.

[5] Y. Ben-Haim and I. Elishakoff, Convex Models of Uncertainty in Applied Mechanics, Elsevier Science, Amsterdam, 1990.

[6] S. Ganzerli, and C. P. Pantelides, "Optimum structural design via convex model superposition," Comput. Struct., vol. 74, pp. 639-647, 2000.

[7] S. S. Rao, and L. Berke, "Analysis of uncertain structural systems using interval analysis," AIAA J., vol. 34, pp. 727-735, 1997.

[8] R. L. Muhanna, and R. L. Mullen, "Uncertainty in mechanics problems interval-based approach," ASCE J. Eng. Mech., vol. 127, pp. 557-566, 2001.

[9] Z. Qui, X. Wang, and J. Chen, "Exact bounds for the static response set of structures with uncertain-but-bounded parameters, "Int. J. Sol. Struct., vol. 43, pp. 6574-6593, 2006.

[10] L. Zadeh, Fuzzy sets", Inf. Control, vol. 8, pp. 338-353, 1965.

[11] S. Valliappan, and T. D. Pham, "Fuzzy logic applied to numerical modeling of engineering problems," Comput. Mech. Adv., vol. 2, pp. 213-281, 1995.

[12] M. D. Munck, D. Moens, W. Desmet, and D. Vandepitte, "A response surface based optimisation algorithm for the calculation of fuzzy envelope FRFs of models with uncertain properties," Comput. Struct., vol. 86, pp. 1080 1092, 2008.

[13] B. Moller, W. Graf, and M. Beer, "Fuzzy structural analysis using level optimization," Comput. Mech., vol. 26, pp. 547-565, 2000.

[14] M. Hanss, "The transformation method for the simulation and analysis of systems with uncertain parameters," Fuzzy Sets Syst., vol. 130, pp. 277-289, 2002.

[15] M. Hanss, Applied Fuzzy Arithmetic: An Introduction with Engineering Applications, Springer-Verlag, Berlin, 2005.

[16] A. Chekri, G. Plessis, B. Lallemand, T. Tison, and P. Level, "Fuzzy behavior of mechanical systems with uncertain boundary conditions," Comput. Methods Appl. Mech. Eng., vol. 189, pp. 863-873, 2000.

[17] A. K. Dhingra, S. S. Rao, and V. Kumar, "Nonlinear membership function in multi-objective fuzzy optimization of mechanical and structural systems," AIAA J, vol. 30, pp. 251-260, 1992.

[18] O. C. Zienkiewicz, The Finite Element Method. Tata McGraw Hill Edition, 1979.

[19] S. S. Rao, and J. P. Sawyer, "Fuzzy finite element approach for the analysis of imprecisely defined systems," AIAA J., vol. 33, pp. 2364-2370, 1995.

[20] W. Verhaeghe, M. D. Munck, W. Desmet, D. Vandepitte, and D. Moens, "A fuzzy finite element analysis technique for structural static analysis based on interval fields.," 4th Int. Workshop Reliable Eng. Comp., pp. 117-128, 2010.

[21] U. O. Akpan, T. S. Koko, I. R. Orisamolu, and B. K. Gallant, "Practical fuzzy finite element analysis of structures," Finite Elem. Anal.Des., vol. 38, pp. 93-111, 2001.
[22] U. O. Akpan, T. S. Koko, I. R. Orisamolu, and B. K. Gallant, "Fuzzy finite element analysis of smart structures," Smart Mater.Struct., vol. 10, pp. 273-284, 2001.

[23] R. L. Muhanna, and R. L. Mullen, "Formulation of fuzzy finite element method for mechanics problems," Comput.-Aided Civil Infrastruct. Eng., vol. 14, pp. 107117, 1999.

[24] M. Hanss, and K. Willner, "A fuzzy arithmetical approach to the solution of finite element problems with uncertain parameters," Mech. Res. Commun., vol. 27, pp. 257-272, 2000

[25] A. S. Balu, and B. N. Rao, "Efficient explicit formulation for practical fuzzy structural analysis," Sadhana, vol. 36, pp. 463-488, 2011.

[26] A. S. Balu, and B. N. Rao, "Explicit fuzzy analysis of systems with imprecise properties, "Int. J. Mech. Mater. Des.," vol. 7, pp. 283-289, 2011.

[27] A. S. Balu, and B. N. Rao, "High dimensional model representation based formulations for fuzzy finite element analysis of structures, "Finite Elem. Anal.Des.," vol. 50, pp. 217-230, 2012.

[28] M. Friedman, M. Ming, and A. Kandel, "Fuzzy linear systems,” Fuzzy Sets Syst., vol. 96, pp. 201-209, 1998.

[29] X. Guo, and Z. Gong, "Block Gaussian elimination methods for fuzzy matrix equations," Int. J. Pure App. Math., vol. 58, pp. 157-168, 2010.

[30] P. Senthilkumar, and G. Rajendran, "New approach to solve symmetric fully fuzzy linear systems," Sadhana, vol. 36, pp. 933-940, 2011.

[31] M. Dehghan, and B. Hashemi, "Solution of the fully fuzzy linear system using the decomposition procedure," Appl. Math. Comput., vol. 182, pp. 1568-1580, 2006.

[32] S. Das, and S. Chakraverty, "Numerical solution of interval and fuzzy system of linear equations," Appl. Appl. Math. : An Int. J., vol. 7, pp. 334-356, 2012.

[33] I. Skalna, M. V., Rama Rao, and A. Pownuk, "Systems of fuzzy equations in structural mechanics," J. Comp. Appl. Math., vol. 218, pp. 149-156, 2008.

[34] D. Behera, D. Datta, and S. Chakraverty, "Development of a finite element solution of a stepped rectangular Bar in presence of fuzziness in material properties," Proc. 5th Int. Conf. Adv. Mech. Eng., SVNIT, Surat-395 007, India, ICAME-2011, pp. 205-209, 2011.

[35] T. J. Ross, Fuzzy Logic with Engineering Applications. John Wiley \& Sons, New York, 2004.

[36] H. J. Zimmermann, Fuzzy Set Theory and its Application. Kluwer academic publishers, London, 2001.

[37] S. S. Bhavikati, Finite Element Analysis. New Age International Publisher, 2005.

[38] S. Chakraverty, and D. Behera, "Fuzzy system of linear equations with crisp coefficients," J. Intell. Fuzzy Syst., vol. 25, pp. 201-207, 2013.

[39] D. Behera and S. Chakraverty, A new method for solving real and complex fuzzy systems of linear equations, Comp. Math. Model., Vol. 23, pp. 507-518, 2012. 